\title{
The Understanding of Temperature Cognition and Thermal Sensation
}

\author{
Jiaolin Wang ${ }^{1}$ \&Wei $\mathrm{Xu}^{1}$ \\ ${ }^{1}$ Guangdong University of Petrochemical Technology \\ NO.139, 2nd Guanshan Road ,Maoming City,Guangdong Province,China 525000
}

\begin{abstract}
KEYWORD: temperature sensitive; sensor; thermal sensation; model
ABSTRACT: Relying on the thermosenstors scattering in the skin, people are capable of adetecting the thermal stimuli to protect themselves from the damage of hazardous environmental factors. Temperature sensitive sensors can be divided into two classes: innocuous thermosensors (warm or cool) and noxious thermonociceptors (hot or cold). Main thermosensors belonged to the transient receptor Potential (TRP) cation channel family. Thermo TRPs respond to distinct thermal thresholds, and their responding mechanism is useful to make the thermal sensation be well understood at the molecular level. Considering that the thermal sensors played vital role in the practical process of temperature perception, some researchers tried to link the temperature traits of thermal sensors with thermal sensation and develop new index for assessing thermal sensation. The evaluation models of thermal sensation that combined the thermosensitive characteristics of sensors with heat transfer occurring in skin tissue were introduced in this paper.
\end{abstract}

\section{Introduction}

Human body tends to keep a constant core temperature through two complementary regulation systems. One system was physiological thermo-regulation system under the autonomic nervous system's control, another system was the central nervous system's control of behavioral thermo-regulation by means of conscious sensations of discomfort (R.J.de Dear\&J.W.R.a.P.F 1993). Based on these research conclusion, sensing exterior thermal stimuli was the first step to regulate the temperature of human body. To survive and adapt to environment better, people must perceive and respond to the change of environment. Temperature perception played a important role in interaction of human body and environment. The painful experience of touching a stove were initiated by somatosensory neurons that innervate human skin. The end of neuron transformed the stimuli into action potential of neuron cell (Lumpkin, E.A \& M.J.Caterina 2007).

The somatosensory neurons scattering in skin were diversity and were broadly classified as $A \beta-, A \delta-$ or C-fibres on the basis of degree of myelination and the speed at which action potentials traveled along afferent fibres(Lumpkin, E.A \&M.J.Caterina;2007). As showed in Figure 1, both A $\beta$ fibers which innervated Merkel cells and $A \beta$ fibers that surrounded hair shafts were thought as touch receptors(Lumpkin, E.A \& M.J.Caterina 2007). A $\delta$-fibers and C-fibers included temperature receptors and nociceptors. A $\delta$-fibers terminated at the dermis. Peptidergic and non-peptidergic C-fibres terminated at different epidermal layers(Zylka et al. 2005). The projection methods to spinal cord were different(Snider et al. 1998). 


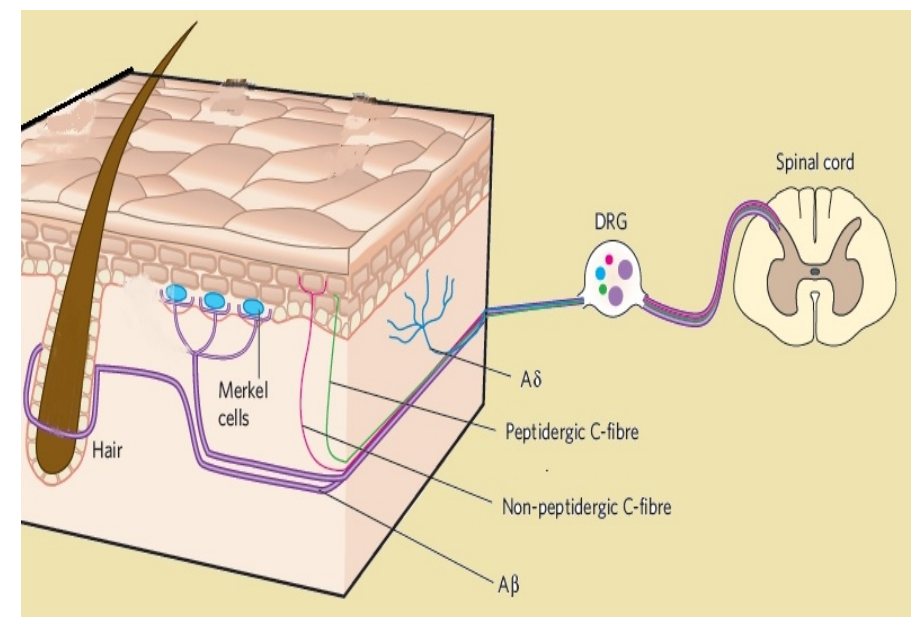

Figure 1. The diversity of somatosensory neurons scattering in skin(Gardner et al. 2000)

Temperature receptors responded to heating or cooling, and mainly contained two kinds of neurons which responded respectively to innocuous and nocuous temperature. The temperature dectection of mammals ranged from $-10^{\circ} \mathrm{C}$ to $60^{\circ} \mathrm{C}$ and different temperature resulted different subjective psychological perception and objective behavioral responses (Lumpkin, E.A. \& M.J.Caterina 2007). Some proofs indicated that the principal temperature sensors of nerve ending of mammls belonged to the transient receptorpotential (TRP) superfamily of cation channels(David E \& Clapham 2003, Voets, T. \& Nilius, B 2003).

\section{Perception of nocuous temperature and pain}

$43^{\circ} \mathrm{C}$ was the threshold at which temperature cognition shift from innocuous warmth to nocuous heat and peole stat to perceive thermal pain(Ardem Patapoutian et al.2003). Nociceptors tranformed heat in ionic current and then induced action potential. Action potential was transmitted to central nerves ending and was transformed into neurotransmitter which was released at frontal end of synapsis. The function of nociceptors depended on cation chennels (M.J. Caterina \& D.Julius 1999, E.W. McCleskey \& M.S. Gold 1999). There were six cation channels involving thermal sensors suchas TRPV1, TRPV2, TRPV3, TRPV4, TRPM8, TRPA1. TRPV1, TRPV2, TRPV3, TRPV4 could be activated by heating, while TRPM8,TRPA1 were activated by cooling. The detective temperature of cation channels ranging from $-10^{\circ} \mathrm{C}$ to $50{ }^{\circ} \mathrm{C}$ corresponded to the physiological temperature range that human can discriminate (ThomasVoets et al.2005). The temperature threshold, at or over this point TRPV1 could be activated, was up to $43^{\circ} \mathrm{C}$, but the temperature activation threshold of TRPV2 was more higher even higher than $52^{\circ} \mathrm{C}$. When temperature was lower than $22^{\circ} \mathrm{C}$, TRPM8 started to be activaed, but if the temperature was lower than $15^{\circ} \mathrm{C}$, TRPA1 was able to detect the nocuous temperature(Magdalene M Moran et al.2004). The activation ranges of TRPV3,TRPV4 were normal temperature. When nociceptors were stimulated by nocuous stimulin, the cation channels opened and ionic current was produced, then action potential was triggered. According to the researchs( Feng Xua et al .2008a, Feng Xua et al. 2008b, Feng Xua,et al .2008c) the model for pain resulted from nocuous heating or cooling could be expressed as follow: first step, nociceptors tranformed the heat of thermal stimulin into electrical signals(nerve impulses); second step, this signals were transmitted to brain in the form of action potential chain; at last, brain decoded the signal and obtained temperature information which was translated into thermal pain. The experiments made the skin of normal initial temperature touch heat source of different constant temperature $\left(50^{\circ} \mathrm{C}, 55^{\circ} \mathrm{C}\right.$, $60^{\circ} \mathrm{C}$ ) 5 seconds and investigated the response of nociceptors located at the depth of $50 \mu \mathrm{m}$. Based on experiments, a model to assesss and predict pain which quantatively expressed pain via the membrane potential had been setted up. The threshold of membrane potential was $-55 \mathrm{mV}$, at or over this point brain sensed thermal pain. 


\section{Perception of innocuous warmth or cold and thermal sensation}

Human sense innocuous temperature relying on thermosensitive neurons scatterring in skin. The charactreristics of temperature reponse of these fibers are shown as Figure 2 (Hensel 1969).

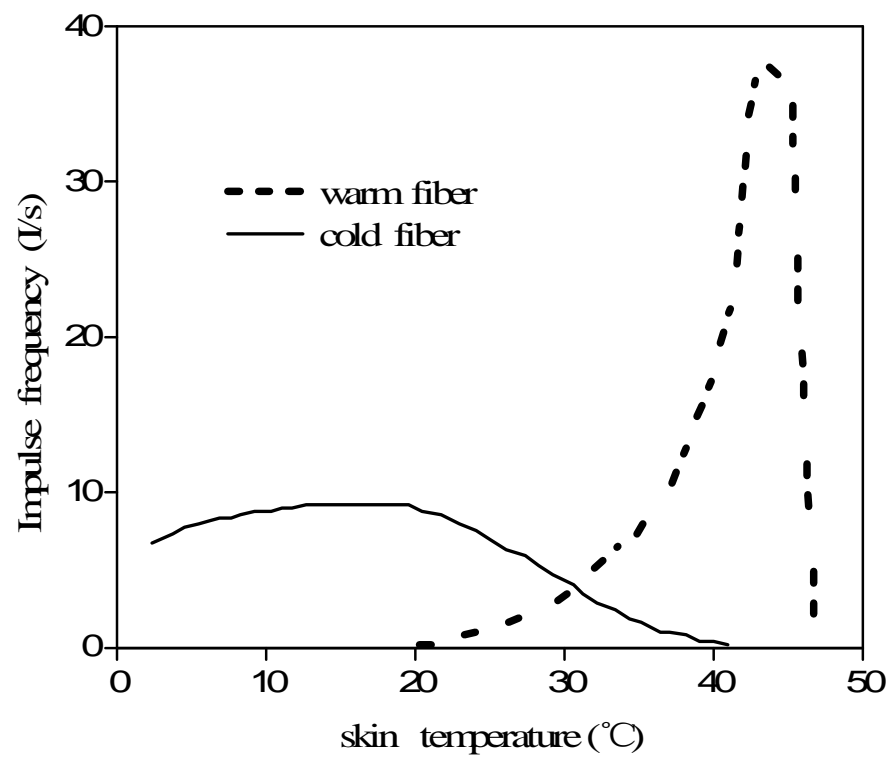

Figure 2. Frequency of individual cold and warm-sensitive fibres in response to skin temperature (Hensel 1969)

The receptors sensitive to cooling were located at the depth of $150-170 \mu \mathrm{m}$, the sensors activated by heating lied at the depth of 300-600 $\mu \mathrm{m}$. Generally speaking, sensors activated by cooling was ten time more than sensors that were sensitive to heatling(Goto et al. 2002). The cold sensitive fibers responded only to cooling, while warm sensitive fibers just reacted to heating, both of the fibers could not repond to mechanical stimulin. The electrophysiology charateristics of thermosensitive fibers were same as traits of TRPV3,TRPV4 which had been identified (ArdemPatapoutian et al. 2003). Hensel (H. HENSEL 1960) identified the static and dynamic thermal characteristics of the thermoreceptors.

Based on these results, Ring and de Dear(R.J.de Dear \& J.W.R.a.P.F 1993) thought human thermal sensation was influenced by temperature of cutaneous thermoreceptors and it's time differential, they developed a thermal response model to assess the thermal sensation in the sudden temperature change condition, and introduced new thermal response index (impulse of the thermoreceptors) which was linear correction with thermal sensation. The model included two sub-models and descibed the process of thermal sensation as following:

(1) Thermal model for temperature response in tissues. This mathematical model expressed how skin temperature changed with environment temperature and time. The environmental thermal stimuli absorbed or released heat, heat transfer between environment and human body losed balance, and at last the skin temperature started to change. Skin temperature was the function of environment temperature and time and the equation linking the two parameters would be acquired according to the law of energy conversation.

(2) The mathematical model linked skin temperature with thermal sensation. When the thermal receptors located in skin are activated by the skin temperature, they fire signals which was transmit to brain ,then people would be aware of the environment and fell warm or cold. So there was a reason to consider that the thermal sensation was a function of skin temperature and it's change rate with time, and a regression model had been obtained.

Later, researchers(Yong-gang Lv \& Jing Liu 2005) developed a thermal comfort model linking thermal comfort with impulses of thermoreceptors. Thermal comfort was the function of impulses of thermoreceptors and expressed as eqution(1) (Yong-gang Lv \& Jing Liu 2005). 
$T C D=\left\{\begin{array}{l}\frac{f-f_{0}}{f_{h l}-f_{0}} \times 15, \text { when } f_{0}<f<f_{h l} \\ -\frac{f-f_{0}}{f_{h l}-f_{0}} \times 15, \text { when } f_{l l}<f<f_{0}\end{array}\right.$

Where TCD is thermal comfort degree, a total degree of 30 is prescribed.TCD $=0$ represents the perfect comfortable point, while TCD $=15$ or -15 are for the hottest and coldest feeling. $\mathrm{f}$ is the frequency of action potential firing at a given thermal environment. Compared with the model of de Dear, the model of Lv was simple, straightforward, and the assessing index responded instantly. Besides the simplicity and fast response time, Lv' model had the potential to be the fundamental model to interpret the relationship between environment and human thermal sensation.

\section{Conclusion}

Up to now, the common available models assessing thermal sensation are based on the heat balance between human and environment. Considering the important role of thermoreceptors which involves with signals transformation and conduction in the practical process of temperature perception, their responses to thermal stimulin should not be ignored any more. The models expressing temperature characteristics of thermosensors have been setted up and can modify the traditional thermal sensation models. The further understanding of temperature perception mechanism at the molecular level makes building a united model which is fit for the assessing both nocuous and innocuous thermal environment be possible.

\section{References}

[1] Ardem Patapoutian, Andrea M. peter, et al (2003). ThermoTRP channels and beyond: mechanisms of temperature sensation. Nature Review Neurosci 4(7): 529-539.

[2] David E. Clapham(2003).TRP channels as cellular sensors. Nature 426, 517-524 .

[3] E.W. McCleskey, M.S. Gold(1999) . Ion channels of nociception. Annual Reviews in Physiology $61835-856$.

[4] Feng Xua, Ting Wena, Keith Seffen a, Tianjian Lub,(2008a). Modeling of skin thermal pain: A preliminary study. Applied Mathematics and Computation 205(1): 37-46.

[5] F. Xua, T.J. Lub, K.A. Seffena (2008b). Skin thermal pain modeling- A holistic method. Journal of Thermal Biology 33(4):223-237.

[6] F. Xua, T.J. Lub, K.A. Seffena (2008c). Biothermomechanics of skin tissues. Journal of the Mechanics and Physics of Solids 56(5):1852-1884.

[7] Gardner, E. P., Martin, J. H. \& Jessell(2000), T. M. in Principles of Neural Science (eds Kandel, E. R, Schwartz, J. H. \& Jessell, T.M.) 430-449.Oxford Univ. Press, New York.

[8] Goto,T, J. Toftum, et al. (2002). Thermal Sensation and Comfort with Transient Metabolic Rates. Proceedings of Indoor Air Conference, June 30 -July 5. USA: Monterey.

[9] Hensel, H. \& Kenshalo, D. R(1969). Warm receptors in the nasal region of cats. J. Physiol. (Lond.) 204, 99-112.

[10]H. HENSEL(1960). A quantitative study of sensitive cutaneous thermoreceptors with C afferrent fibres ,Physiol.

[11]Lumpkin, E.A. and M.J.Caterina (2007). Mechanisms of sensory transduction in the skin. Nature 445(7130):858-865.

[12] Magdalene M Moran, Haoxing Xu and David E Clapham (2004). TRP ion channels in the nervous system. Current Opinion in Neurobiology 14(3): 362-369.

[13]M.J. Caterina, D.Julius(1999). Sense and specificity: a molecular identity for nociceptors. Current Opinion in Neurobiology 9: 525-530. 
[14]R.J.de Dear, J.W.R.a.P.F. (1993). Thermal Sensations Resulting from Sudden Ambient Temperature Changes. indoor air 3:181-192.

[15]Snider, W. D. \&McMahon, S. B(1998). Tackling pain at the source: new ideas about nociceptors. Neuron 20, 629-632.

[16]Thomas Voets, Karel Talavera, Grzegorz Owsianik \& Bernd Nilius (2005). Sensing with TRP channels. NatureBiology.

[17] Voets, T. \& Nilius, B(2003). TRPs make sense. J. Membr. Biol. 192, 1-8 .

[18] Yong-gang Lv, Jing Liu(2005). Interpretation on thermal comfort mechanisms of human bodies by combining Hodgkin-Huxley neuron model and Pennes bioheat equation. Forschung im Ingenieurwesen 69:101-114.

[19]Zylka, M. J., Rice, F. L. \& Anderson (2005), D. J. Topographically distinct epidermal nociceptive circuits revealed by axonaltracers targeted toMrgprd. Neuron 45, 17-25. 